\title{
Correction to: Additional value of metabolic parameters to PET/CT-based radiomics nomogram in predicting lymphovascular invasion and outcome in lung adenocarcinoma
}

Pei Nie ${ }^{1} \cdot$ Guangjie Yang ${ }^{2} \cdot$ Ning Wang $^{3} \cdot$ Lei Yan ${ }^{2} \cdot$ Wenjie Miao ${ }^{2} \cdot$ Yanli Duan ${ }^{2} \cdot$ Yanli Wang $^{4} \cdot$ Aidi Gong $^{2}$.

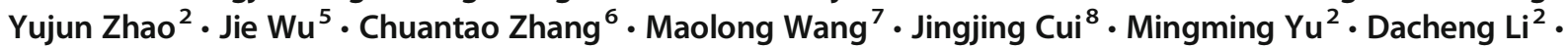
Yanqin Sun $^{2} \cdot$ Yangyang Wang ${ }^{2}$ - Zhenguang Wang ${ }^{2}$

Published online: 28 July 2020

(C) Springer-Verlag GmbH Germany, part of Springer Nature 2020

\section{Correction to: European Journal of Nuclear Medicine and} Molecular Imaging. https://doi.org/10.1007/s00259-020-04747-5

Figures 1 and 4 are incorrect in the original manuscript. The correct Figs. 1 and 4 are shown below.

Also, In Table 4, "EGFR mutation ( \pm ND)" is not correct, it should be "EGFR mutation (+/-/ ND)", because "+"” and "-"” means positive and negative, respectively. The correct Table 4 is shown below:

The original article has been corrected.

This article is part of the Topical Collection on Erratum

The online version of the original article can be found at https://doi.org/ $10.1007 / \mathrm{s} 00259-020-04747-5$

Zhenguang Wang

doctorwzg2002@hotmail.com

1 Department of Radiology, The Affiliated Hospital of Qingdao University, No. 16 Jiangsu Road, Qingdao, Shandong, China

2 Department of Nuclear Medicine, TheAffiliated Hospital of Qingdao University, No. 59 Haier Road, Qingdao, Shandong, China

3 Department of Radiology, Shandong Provincial Hospital, No. 324, Jingwu Road, Jinan, Shandong, China

4 PET-CT Center, Qingdao Central Hospital, No. 127 Siliu South Road, Qingdao, Shandong, China
Department of Pathology, The Affiliated Hospital of Qingdao University, No. 16 Jiangsu Road, Qingdao, Shandong, China

6 Department of Oncology, The Affiliated Hospital of Qingdao University, No. 16 Jiangsu Road, Qingdao, Shandong, China

7 Department of Thoracic Surgery, The Affiliated Hospital of Qingdao University, No. 16 Jiangsu Road, Qingdao, Shandong, China

8 Huiying Medical Technology Co., Ltd, No. 66 Xixiaokou Road, Beijing, China 

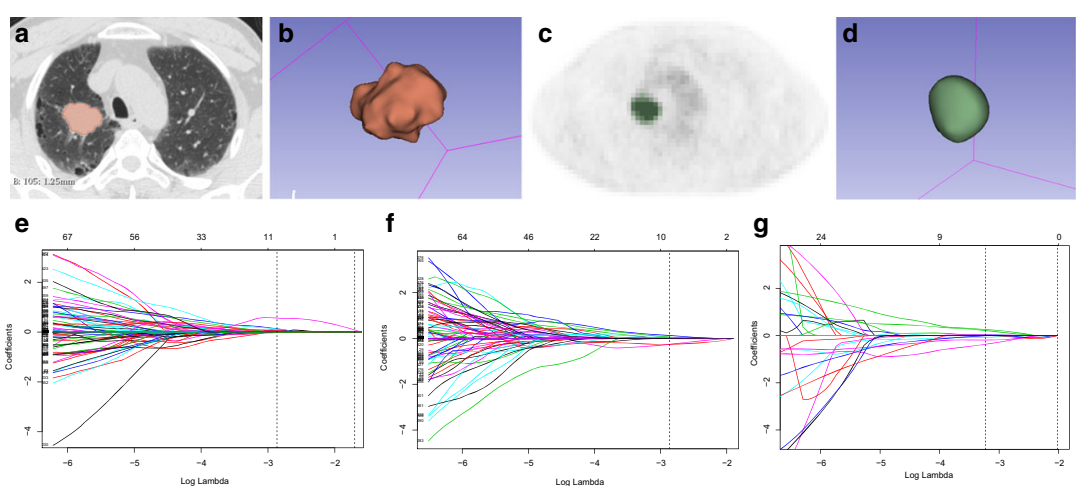

f

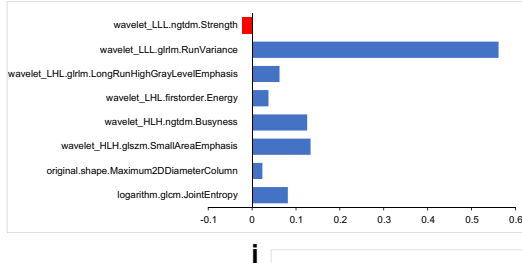

j

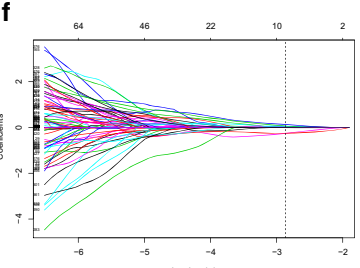

g

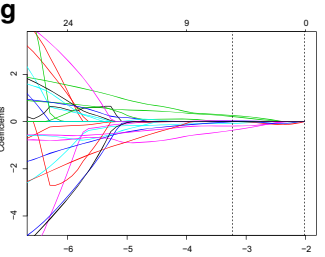

i

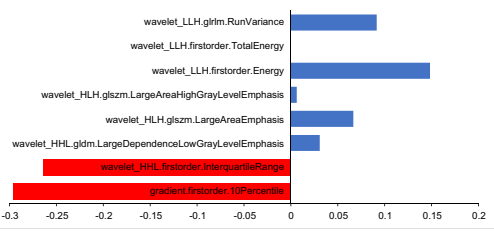

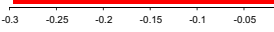

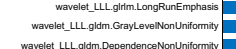

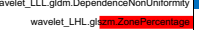

wavelet_HLH.ngttm.Contrast

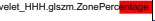

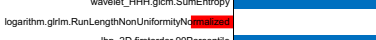

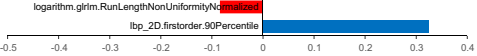

$\mathbf{k}$
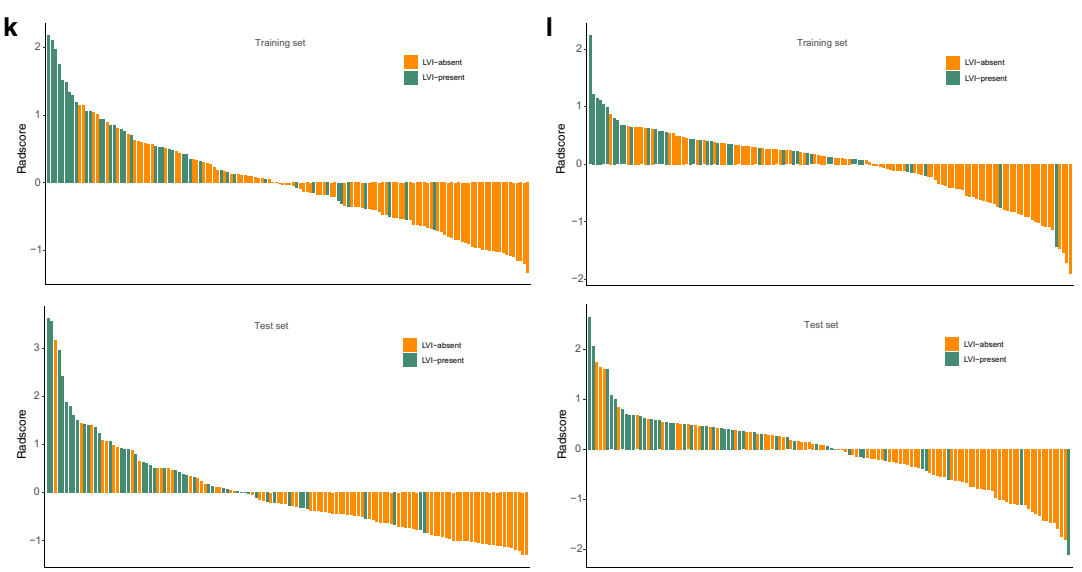

m
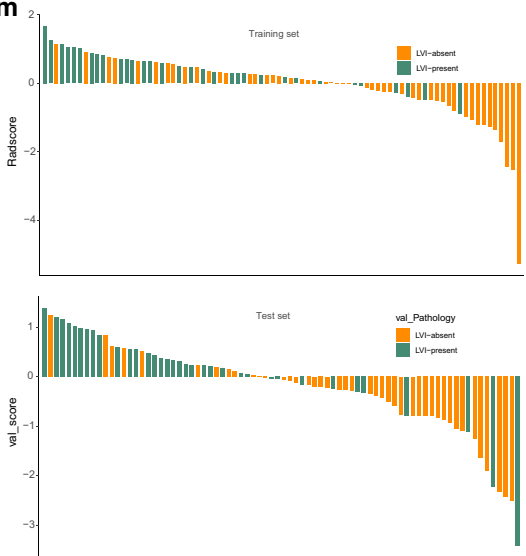

Fig. 1 Construction of radiomics signatures (RSs). a, b CT ROI segmentation. c, d PET ROI segmentation. e, f, g Two-dimensional (2D) CT, three-dimensional (3D) CT, and PET features selection using the least absolute shrinkage and selection operator (LASSO) regularization. $\mathbf{h}, \mathbf{i}, \mathbf{j}$ The selected 8 2D CT features, 8 3D CT features, 10 PET features and their coefficients. $\mathbf{k}, \mathbf{l}, \mathbf{m}$ The 2D CT radiomics scores (Rad-scores), 3D CT Rad-scores, and PET Rad-scores for each patient in the training and test sets 


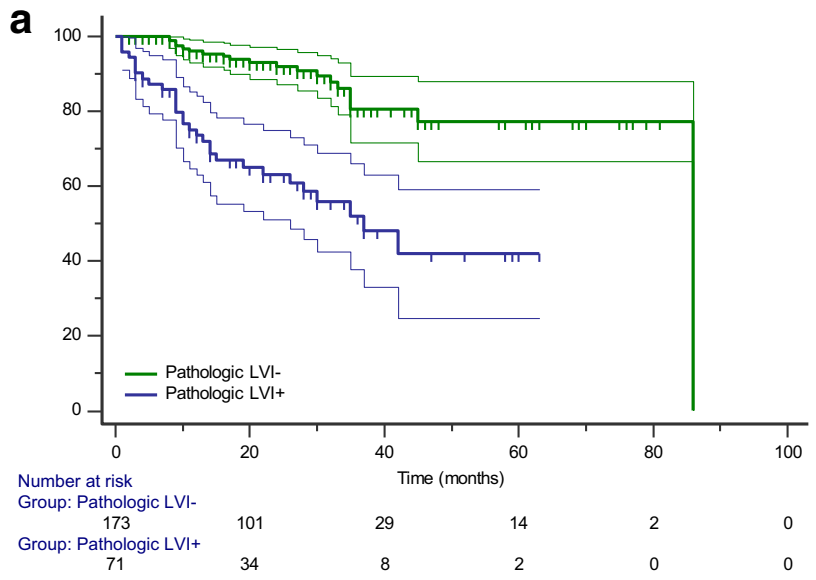

C

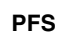

Overall

CA125 > $12.95 \mathrm{U} / \mathrm{ml}$

CEA $>1.81 \mathrm{ng} / \mathrm{ml}$

NSE > $13.71 \mathrm{U} / \mathrm{ml}$

Pathologic LVI +

High-risk histologic subtype

SUVmax $>5$

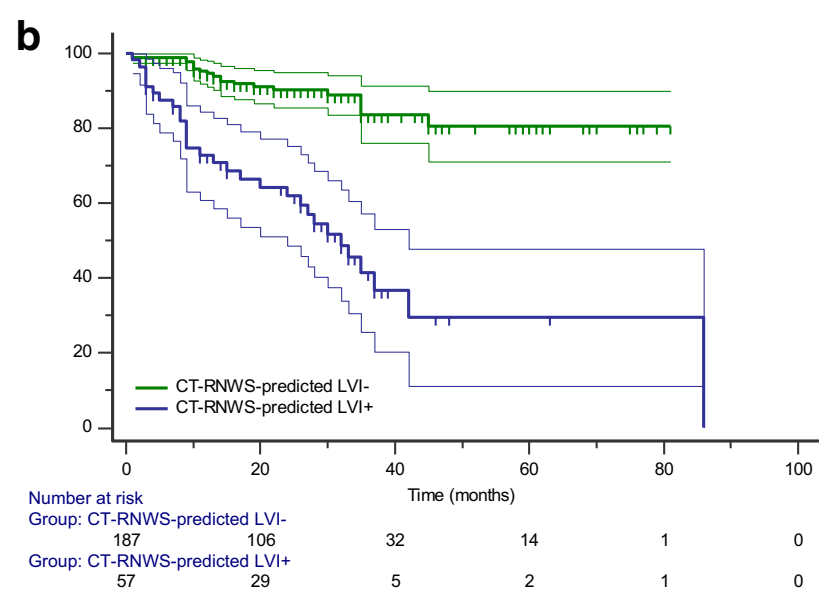

HR(95\%Cl)

2.356(1.152,4.819)

4.734(1.099,20.381)

2.394(1.143,5.013)

2.398(1.168,4.923)

2.526(1.012,6.303)

$3.032(1.189,7.730)$

Fig. 4 Progression-free survival (PFS) curves according to pathologic LVI status (a) and CT-RNWS-predicted LVI status (b) in the 244 CTRNWS-predicted cohort. $\mathbf{c}$ Forest plot of independent predictors of PFS with a multivariate Cox regression model. LVI, lymphovascular invasion;
RNWS, radiomics nomogram with SUVmax; HR, hazard ratio; CI, confidence interval; CA125, carbohydrate antigen 125; CEA, carcinoembryonic antigen; NSE, neuron-specific enolase
Table 4 The pathologic and therapeutic factors

\begin{tabular}{lll}
\hline Factors & Pathology LVI+ $(\boldsymbol{n}=\mathbf{8 7})$ & Pathology LVI- $(\boldsymbol{n}=\mathbf{1 8 5})$ \\
\hline Stage & & \\
I & 25 & 134 \\
II & 20 & 18 \\
III & 37 & 26 \\
IV & 5 & 7 \\
Histological subtype (Low risk/ High risk) & $18 / 69$ & $121 / 64$ \\
EGFR mutation (+/-/ ND) & $29 / 25 / 33$ & $88 / 48 / 49$ \\
Surgical approach (Lobectomy/ Sublobectomy) & $80 / 7$ & $169 / 16$ \\
Treatment after surgery & & \\
Chemotherapy & 31 & 32 \\
Targeted therapy & 4 & 16 \\
Chemotherapy and targeted therapy & 11 & 8 \\
No treatment & 41 & 129 \\
\hline
\end{tabular}

LVI (lymphovascular invasion); EGFR (epidermal growth factor receptor); ND (not detected).

Publisher's note Springer Nature remains neutral with regard to jurisdictional claims in published maps and institutional affiliations. 